\title{
EFICIÊNCIA DA GONADOTROFINA CORIÔNICA EQÜINA (eCG) E DO DESMAME TEMPORÁRIO (DT) EM PROTOCOLOS PARA A INSEMINAÇÃO ARTIFICIAL EM TEMPO FIXO (IATF) EM VACAS NELORE, PREVIAMENTE TRATADAS COM PROGESTERONA(P4) E BENZOATO DE ESTRADIOL (BE).
}

\author{
Andre Luis Bastos Souza*, Luiz Ernandes Kozicki, João Filipi Scheffer Pereira, \\ Márcio Saporski Segui, Romildo Romualdo Weiss, Melina Andrea Formighieri Bertol \\ *Correspondência: andrebastos@vetmaxi.com:
}

RESUMO: O objetivo do estudo foi checar a eficiência reprodutiva da eCG e do desmame temporário em protocolos para IATF em vacas Nelore pluríparas, previamente tratadas com progesterona intravaginal e benzoato de estradiol (BE). Foram utilizadas 343 animais, com ECC médio de 2,65 (1=magra; $5=$ gorda), no período pós parto (media 51,9 dias), e divididas em dois grupos(G): $\mathrm{GeCG}$ (grupo gonadotrofina coriônica equina; $n=144$ ) recebeu no dia 0 (d0) 0,558 g de P4 + 1,0 mg de BE; em d8 (remoção da P4 + 0,075 mg de PGF2 alfa+1,5 mg de cipionato de estradiol-ECP + 400UI de eCG); em d10 execução da IATF; o GDT (grupo desmame temporário; $n=199$ ) o mesmo que GeCG sem eCG no d8, e com desmame temporário por 48 horas e retorno dos bezerros às mães no dia da IATF(d10). A taxa de prenhes (TP) à IATF, após 35 dias resultou em 59,0 e 63,3\% respectivamente para os GeCG e GDT $(P>0,05)$ e a TP à IATF + 0 repasse com touros, em 90,9 e $95,9 \%(P=0,056)$, após 90 dias de estação de monta. Concluiuse que ambos os protocolos mostraram-se eficientes em fazendas comerciais de bovinos de corte, enfatizando-se a utilização do DT onde haja facilidade de manejo. Palavras-chave: Vacas de corte; IATF; Progesterona longa ação; eCG; Desmame Temporário 


\title{
EFFICIENCY OF EQUINE CHORIONIC GONADOTROPHIN (ECG) AND TEMPORARY WEANING (TW) BY PROTOCOLS FOR TIMED-ARTIFICIAL INSEMINATION (TAI) IN NELLORE COWS, PREVIOUSLY SUBJECTED TO PROGESTERONE (P4) AND ESTRADIOL BENZOATE (EB).
}

\begin{abstract}
The aim of the study was to check the reproductive efficiency by eCG and temporary weaning use for TAl in multiparous Nellore cows, after previous treatment with intravaginal progesterone and estradiol benzoate (EB). Three hundred and forty three animals were used, presenting BCS average 2.65 (1=thin; $5=$ obese), in the post-partum period (mean $=51.9$ days), and assigned into two groups $(\mathrm{G})$ : $\mathrm{GeCG}$ (equine chorionic gonadotropin group; $n=144$ ) received on day 0 (d0) $0.558 \mathrm{~g} \mathrm{P4}+1.0 \mathrm{mg}$ EB; in d8 (P4 removal + 0,075mg PGF2alpha + $1.5 \mathrm{mg}$ of $\mathrm{ECP}+400 \mathrm{IU}$ of eCG); in d10 TAl was performed; the GTW (temporary weaning group; $n=199$ ) the same as GeCG without eCG on d8, plus TW for 48 hours and return of calves to dams in the TAI day (d10). TAI pregnancy rate (PR), after 35 days $A I$, resulted in 59.0 and $63.3 \%$ respectively for the GeCG and GTW (P > 0.05); the $\mathrm{PR}$ related to TAI + natural service (bulls), after 90 days breeding season resulted in 90.9 and $95.9 \%(P=0.056)$. It was concluded, both protocols were effective in commercial farms beef cattle, emphasizing the use of TW in farms, where the animal management become more favorable.
\end{abstract}

Key Words: Beef cattle; TAI; Progesteron device; eCG; Temporary weaning 


\section{INTRODUÇÃO}

Os ganhos genéticos que a inseminação artificial (IA) proporciona aos rebanhos bovinos (corte e leite), a credenciam como uma das mais utilizadas no mundo todo. Muito embora a IA no Brasil ainda seja timidamente utilizada, houve expansão nos últimos dez anos, passando de 5,0 \% em 2002 para 10,0\% em 2012 (BARUSELLI et al., 2012). Esse percentual foi em parte alavancado, pela expansão da Inseminação artificial em tempo fixo (IATF), reportada em relatório da ASBIA (2014), dada a comercialização de cerca de 13 milhões de doses de sêmen bovino no Brasil. Essa expansão foi semelhantemente acompanhada por significativos progressos no desenvolvimento de protocolos para IATF, sobretudo na pecuária de corte brasileira.

Neste contexto, e dentre muitas variações dos protocolos utilizados por estudiosos da área, o uso da eCG e do desmame temporário (DT) foi paralelamente pesquisado. A utilização da eCG e do DT no dia 8 do protocolo em bovinos, associados a tratamento hormonal prévio com dispositivo intravaginal com progestágeno (P4) e $E 2$, tornaram-se ferramentas valiosas na melhoria da TP (CUTAIA e BO, 2012).

$\mathrm{Na}$ América do Sul um dos protocolos mais utilizados para a IATF é a associação de P4 com E2 em função do feedback negativo exercido no eixo hipotalâmico-hipofisário, com redução da secreção de $\mathrm{FSH}$ e $\mathrm{LH}$, além de, adicionalmente, conduzir à atresia folicular (BURKE et al., 2003; BARUSELLI et al., 2014).

Contudo os tratamentos nesta direção progrediram, e estudos foram feitos com a eCG em vacas em anestro no momento da remoção da fonte de P4 (BARUSELLI et al., 2004). A utilização de 400 Ul de eCG à remoção da P4 intravaginal resultou em aumento da concentração plasmática de $\mathrm{P} 4$, assim como as TP em vacas com bezerro-aopé no anestro pós parto. (BARUSELLI et al., 2004; BO et al., 2007). Sá Filho et al. (2010) utilizaram eCG na remoção do implante auricular de norgestomet (P4), e verificaram aumento da TP em vacas Bos indicus, dentre outros parâmetros reprodutivos testados.

Barreiros et al. (2014), investigaram três grupos de vacas Nelore em anestro, previamente tratadas com $\mathrm{P} 4$, sendo um controle (=sem eCG e sem desmame temporário - DT), outro grupo com eCG e o terceiro com DT após a remoção da P4. Eles verificaram que tanto a administração da eCG como o DT haviam melhorado a fertilidade, constatando que 0 DT adicionalmente, aumentara a concentração de P4 no plasma. Edwards (1985) havia constatado que o DT aumentava a concentração e os pulsos de LH depois de 48 a 56 horas, após remoção do bezerro, enfatizando este efeito mormente em animais sem ciclicidade ovariana.

Sá Filho et al. (2009), avaliaram os efeitos do DT, da eCG e do FSH em protocolos de IATF em vacas de corte, concluindo que os tratamentos com DT ou com eCG incrementavam consistentemente a TP à IATF, sendo contudo, o tratamento com FSH menos consistente que os demais. Ereno et al.(2007) pesquisaram vacas Nelore avaliando o DT e aplicação de eCG em protocolos de IATF. Eles verificaram TP de 50,$5 ; 53,5$ e $54,7 \%$ respectivamente para os grupos IATF, IATF + DT e IATF + eCG, concluindo que a associação do DT pós tratamento com P4 intravaginal, aumentava as TP, em contraposição ao uso da eCG + tratamento com P4, não elevando a TP nas vacas ciclando e com boa condição corporal. Neste contexto denota-se que há ainda pontos a serem elucidados no tocante à utilização de eCG ou do DT sobre a fertilidade de vacas de corte. Deste 
modo hipotetizou-se no presente estudo que o DT poderia deter potencial na melhoria da TP em vacas de corte, ainda no período pós-puerperal precoce, se comparado a tratamentos com a eCG.

O estudo objetivou verificar a eficiência reprodutiva da eCG e do DT de bezerros por 48 horas seguidas em protocolos de IATF, após prévio tratamento com P4 intravaginal e BE em vacas de corte Bos taurus indicus.

\section{MATERIAL E MÉTODOS}

No estudo foram utilizadas 343 vacas da raça Nelore oriundas de fazenda comercial, dentro de um período médio de serviço de 51,9 dias (40 a 69), escore da condição corporal (ECC) médio de 2,65 (escala 1= magra; $5=$ gorda), havendo $60,0 \%$ dos animais em anestro ao início do estudo e 40,0\% ciclando, verificadas por ultrasssonografia ovariana (ultrassom modelo Tringa, Japan). Os animais tinham acesso livre à pastagem (Brachiaria decumbens), ao sal mineral e água ad libitum. As vacas foram previamente submetidas ao escaneamento de ovários para verificar a condição de ciclicidade ovariana (presença de CL ou de folículos > que 8 $\mathrm{mm}$ de diâmetro) ou de anestro (ausência de CL ou folículos < que 8 $\mathrm{mm})$. Dois grupos de vacas foram constituídos: o grupo gonadotrofina coriônica equina (GeCG; $n=144$ ) e o grupo desmame temporário (GDT; $\mathrm{n}=199$ ). Ambos foram submetidos aos seguintes tratamentos: GeCG recebeu no dia 0 (d0) 0,558 g de P4 intravaginal (Cronipress monodose, Biogenesis Bagó, Curitiba) + 1,0 mg de BE IM (Cronibest, Biogenesis Bagó); no d8 remoção da P4 + PGF2 alfa IM (0,075 $\mathrm{mg}$ de D-cloprostenol, Croniben, Biogenesis Bagó) + 1,5 mg de cipionato de estradiol (ECP) IM (Zoetis, SP) + $400 U I$ de gonadotrofina coriônica eqüina
(eCG) IM (Folligon, MSD, São Paulo); no d10 procedeu-se à IATF; o GDT (grupo desmame temporário; $n=199$ ) o mesmo que o GeCG sem eCG no d8, mas com desmame temporário por 48 horas seguidas e retorno dos bezerros às mães no d10 (=dia da IATF) (esquemas dos protocolos abaixo).

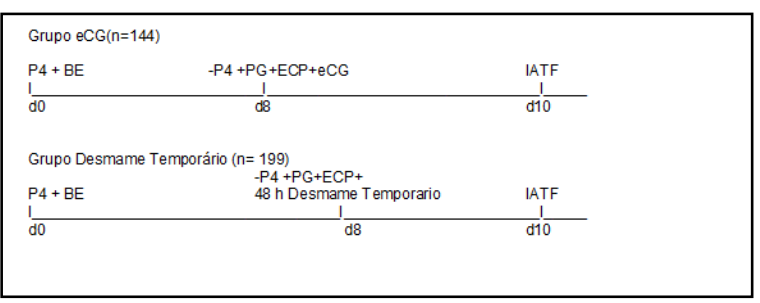

\section{ANÁLISE ESTATISTICA}

Dados da TP dos grupos foram analisados utilizando-se a ANOVA e o teste exato de Fisher, mediante o soft GraphPad Prism, versão 5.0 (Prism 5 Statistics Guide, 2007, GraphPad Software INC., San Diego, CA - USA), visando quantificar os níveis de diferença entre os grupos. As diferenças entre as TP foram calculadas pelo teste exato de Fisher ao nível de $\mathrm{P}<0,05$.

\section{RESULTADOS E DISCUSSÃO}

Os dados gerados encontram-se na Tabela 1. Observa-se os resultados da TP derivados da IATF, da IATF somados aos do repasse com touros durante a estação de monta. Na mesma tabela dados percentuais de animais cíclicos e os em anestro podem ser vistos. 
Tabela 1 - Vacas Nelore previamente submetidas a protocolo de IATF com progesterona intravaginal e prostaglandina F2alfa e tratadas com 400 Ul de eCG ou com desmame temporário (DT) por 48 horas. $(n=343)$.

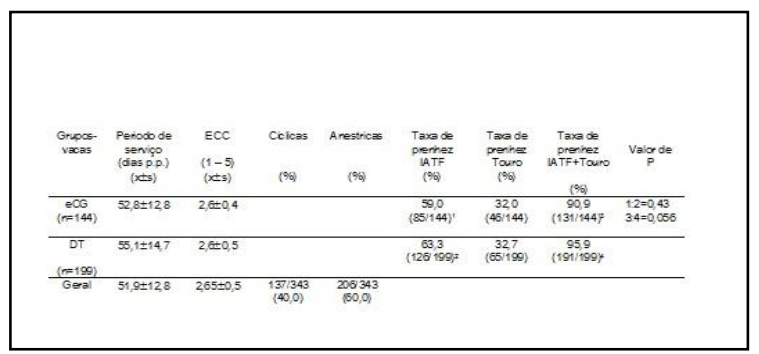

Diversos estudos na área reprodutiva de bovinos de corte, tem sido conduzido, visando elevar a eficiência dos protocolos hormonais para a IATF. Barreiros et al. (2014), empregaram a eCG ou o DT após a remoção da $\mathrm{P} 4$ longa ação. No presente estudo comparou-se protocolos hormonais incluindo a administração de eCG ou DT após tratamento prévio com P4 por 8 dias. As TP originadas das IATFs resultaram em 59,0\% e 63,3\% respectivamente para os GeCG e GDT $(P=0,43)$, e as TP somadas $(=$ IATF + repasse de touros) resultaram em $90,9 \mathrm{e}$ $95,9 \%(P=0,056)$. Observa-se que não houve significância em ambas as variáveis, porém o GDT mostrou-se superior ao final da estação de monta (tendência), certificando o DT como um manejo válido, e que pode ser adotado em fazendas comerciais brasileiras produtoras de bovinos de corte. $\mathrm{Na}$ presente pesquisa não se fez estudos e cálculos de custos diferenciais da adoção dos protocolos hormonais, porém a TP do GDT configurou-se adicionalmente como uma robusta ferramenta para a melhoria da eficiência reprodutiva em vacas com bezerro-aopé (CUTAIA e BO, 2012).

Sá Filho et al. (2009), relatam que o uso de $400 \mathrm{Ul}$ de eCG ou do DT consistentemente melhoraram a TP na
IATF em vacas Nelore. No presente estudo as TP para os GeCG e GDT foram mais elevadas (respectivamente 59,0 e 63,3 - tabela 1) que as reportadas por Sá Filho et al. (2009) $(54,3$ e 51,5$)$, ou as por Ereno et al. (2007) $(54,8$ e 53,65\%), ao trabalharem com vacas Nelore. Contrariamente ao que ocorreu no presente estudo, Ereno et al.(2007) obtiveram percentuais de TP nos animais tratados com eCG, pouco superiores (sem significância) ao se comparar com o grupo DT.

O uso de eCG tem demonstrado eficiência ao desenvolver folículos em vacas de corte em função das relações com FSH e LH (YAVAS e WALTON, 2000), assim como tem proporcionado aumento do diâmetro do folículo dominante, incrementando a taxa de ovulação em animais sem ciclicidade (SÁ FILHO et al., 2005). O comprimento da meia vida do eCG é longa (CARRUTHERS, 1986) e tem a capacidade de se ligar a receptores de LH e FSH (MURPHY e MARTINUK, 1991), estimulando o crescimento da teca interna e da camada da granulosa do folículo dominante (KURAN et al., 1996). O eCG potencialmente incrementa o desenvolvimento folicular, assim como favorece 0 ambiente endócrino durante 0 proestro (maior concentração de E2) e no diestro (maior concentração de $\mathrm{P} 4$ ), proporcionando aumento da fertilidade (SÁ FILHO et al., 2009). Por outro lado o DT (retirada de bezerros por 48 a $96 \mathrm{~h}$ ) favorece 0 aumento da frequência de pulsos de $\mathrm{LH}$ (DUNLAP et al., 1981, SHIVELY e WILLIAMS, 1989, WILLIAMS e GRIFFITH, 1995), e a quantidade de receptores foliculares para $\mathrm{LH}$ e $\mathrm{FSH}$ (Walters et al., 1982), otimizando ações à ovulação. No período pós parto em bovinos de corte, há na realidade, forte redução da pulsatilidade de $\mathrm{LH}$, em função da depleção das concentrações do hormônio na adenohipófise (NETT et al.,1987). O afastamento temporário do 
bezerro da mãe acarreta aumento das concentrações e dos pulsos de LH num breve período de 48 a 56 horas após. $\mathrm{O}$ ato da mamada inibe a liberação de LH hipofisário, assim como ocasiona redução do número de pulsos de $\mathrm{LH}$, de modo que 8 horas após o retorno do bezerro à mãe, as concentrações e a frequência de pulsos de LH decrescem substancialmente (EDWARDS,1984). Vacas amamentando e sem ciclicidade secretam LH de modo pulsátil, iniciando esse mecanismo, usualmente na $3 a$ e 4 semanas pós parto, sendo os pulsos tipicamente de baixa amplitude e baixa frequência, refletindo reduzida liberação de $\mathrm{GnRH}$ hipotalâmico (CARRUTHERS e HAFS, 1980; PETERS et al., 1981). O DT tem sido utilizado para aumentar os níveis de $\mathrm{GnRH}$ e $\mathrm{LH}$ incrementando a taxa de ovulação em vacas em anestro mesmo sem tratamento hormonal (GAZAL et al.,1998). O DT claramente estimula adequadas secreções de $\mathrm{LH}$, de modo que, um suporte adicional de gonadotrofina, parece não ser necessário para o desenvolvimento final do folículo (SÁ FILHO et al., 2009). Isto posto, concluiu-se que ambos os protocolos foram eficientes ao final da estação de monta, realçando-se entretanto, que o DT mostrou-se ainda mais eficiente que a eCG evidenciada pela TP final de cada grupo; sugere-se outros estudos sobre o DT em bovinos de corte, visando dentre outros pontos, observar não só aspectos endócrinos como econômicos em programas de IATF.

\section{REFERÊNCIAS}

ASBIA - Associação Brasileira de Inseminação Artificial - 2014.

BARREIROS,T.R.R.;

BLASCHI,W.; SANTOS,G.M.G.;

ANDRADE, E.R.; BARUSELLI,P.S.; SENEDA,M.M. Dynamics of follicular growth and progesterone concentration in cyclic and anestrus suckling Nelore cows (Bos indicus) treated with progesterone, equine chorionic gonadotropin, or temporary calf removal. Theriogenology. v.81,p.651-656,2014.

BARUSELLI, P. S.; MADUREIRA, E. H.; MARQUES, M.O.; RODRIGUES, C.A.; NASSER, L. F.T.; SILVA, R.C P; REIS, E.L.; SÁ FILHO, M.F. Efeito do tratamento com eCG na taxa de concepção de vacas Nelore com diferentes escores de condição corporal inseminadas em tempo fixo (Análise retrospectiva). In: XVIII Reunião Anual da Sociedade Brasileira de Transferência de Embriões, 2004, Barra Bonita. Acta Scientiae Veterinariae. v. 32. p. 228, 2004.

BARUSELLI, P.S.; MARQUES, M.O.; RIBEIRO JUNIOR, M.; SILVA, R.C.P.; VIEIRA, L.M.; SÁ FILHO, M.F. Como otimizar a eficiência reprodutiva de programas de inseminação artificial e de transferência de embriões em bovinos: reprodução de precisão. 6. Simpósio Internacional de Reprodução Animal Aplicada. 07 e 08 de agosto de 2014, Londrina - PR, p. 76-100, 2014.

BARUSELLI, P.S.; REIS, E.L.; MARQUES, M.O.; NASSER, L.F.; BO, G.A. The use of treatments to improve reproductive performance of anestrus beef cattle in tropical climates. Animal Reproduction Science. V.82-83, p. 479486, 2004.

BARUSELLI,P.S.; SALES,J.N.S.; SALA,R.V.; VIEIRA,L.M.; SÁ FILHO,M.F. History, evolution and perspectives of timed artificial insemination programs in Brazil. Animal Reproduction. v.9, p.139-152, 2012.

BO, G.A.; CUTAIA, L.E.; SOUZA, A.H.; BARUSELLI, P.S. Systematic reproductive management in dairy herds. New Zealand Veterinary Association (NSVA). Conference 2007. Dairy cattle Conference. Christchurch, New Zealand. P.155-168. (disponible on line eSciQuest). 
Eficiência da gonadotrofina coriônica eqüina (ecg) e do...

BURKE, C.R.; MUSSARD, M.L.; GASSER,C.L.; GRUM, D.E.; DAY, M.L. Estradiol benzoate deals new folicular wave emergence in a dose-dependent manner after ablation of the dominant ovarian follicle in cattle. Theriogenology. v.60, p. 647-658, 2003.

CARRUTHERS, T.D.; HAFS, H.D. Suckling and four times daily milking: influence on ovulation, estrus and sérum luteinizing hormone, glucocorticoids and prolactina postpartum. Journal of Dairy Science. v. 50, p.919-925, 1980.

CARRUTHERS, T.D. Principles of hormone therapy in theriogenology. In: MORROW, D.A. Current therapy in theriogenology 2. Diagnosis, treatment and prevention of reproductive diseases in small and large animals. WB Sauders, 1986, p.4. 1986.

CUTAIA, L.; BO, G.A. Uso de la eCG a programas reproductivos en ganado bovino. 5. Simposio Internacional de Reprodução Animal Aplicada. 5 a 6 de otubro de 2012, Londrina - PR, p. 109135, 2012.

DUNLAP, S.E.; KISER, T.E.; COX, N.M. Cortisol and luteinizing hormone after adrenocorticortropic hormone administration to postpartum beef cows. Journal of Animal Science. v.52, p.587593, 1981.

EDWARDS, S. The effectsof short term calf removal on pulsatile LH secretion in the postpartum beef cow. Theriogenology. V.23,p.777-785, 1985.

ERENO, R.L.; BARREIROS, T.R.R.; SENEDA, M.M.; BARUSELLI, P.S.; PEGORER, M.F.; BARROS, C.M. Taxa de prenhez de vacas Nelore lactantes tratadas com progesterona associada à remoção temporária de bezerros ou aplicação de gonadotrofina coriônica eqüina. Revista Brasileira de Zootecnia. v.36, n.5, p. 1288-1294, 2007.

GAZAL,O.S.; STANKI,R.L.;
LESHIN,L.S.; THOMAS, M.G.;
KEISLER, D.H.; ANDERSON, L.L. Gonadotropin-releasing hormone secretion into third-ventricle cerebrospinal fluid $f$ cattle: correspondence with the tonic and surge release of luteinizing hormone and its tonis inhibition by suckling and neuropeptide $Y$. Biology of Reproduction. v.59, p.676-683, 1998.

KURAN, M.; HUTCHINSON, J.S.M.; BROADBENT, P.J. The responce of bovine granulosa cells to diferente gonadotropins in culture. Animal Reproduction Science. v.45, p.1-2, 1996.

MURPHY, B.D.; MARTINUK, S.D. Equine chorionic gonadotropin. Endocrinology Review. v.12, p.13051319, 1991.

NETT, T.M.; CERMAK, D.; BRADEN, T. Pituitary receptors for $\mathrm{GnRH}$ and estradiol, and pituitary contente of gonadotropis in beef cows. I changes during the estrous cycle. Domestic Animal Endocrinology. v.4, p. 123-132, 1987.

PETERS, A.R.; LAMMING, G.E.; FISCHER, M.W. A comparison of plasma LH concentrations in milked and suckling postpartum cows. Journal of Reproduction and Fertility. v.60, p. 567573, 1981.

SÁ FILHO, M.F.; AYRES,H.; FERREIRA, R.M.; MARQUES,M.O.; REIS,E.L.; SILVA, R.C.P.; RODRIGUES,C.A.; MADUREIRA,E.H.; BO,G.A.; BARUSELLI, P.S. Equine chorionic gonadotropin-releasing hormone enhance fertility in a norgestomet-based, timed artificial insemination protocol in suckled Neloe ( Bos indicus) cows. Theriogenology. v.73,p. 651-658,2010.

SA FILHO, M.F.; PENTEADO, L.; REIS, E.L.; GIMENES, L.U.; BARUSELLI, P.S. Efeito da ciclicidade e do tratamento com eCG na dinâmica folicular e na taxa 
de concepção de novilhas Nelore tratadas com implante auricular de norgestomete benzoato de estradiol. Acta Scientiae Veterinariae. v.33, p.265, 2005 (abstract).

SÁ FILHO, O.G.; MENEGHETTI, M.; PERES, R.F.G.; LAMB,G.C.; VASCONCELOS,J.L.M. Fixed-time artificial insemination with estradiol and progesterone for Bos indicus cows II: Strategies and factors affecting fertility. Theriogenology. v.72, p. 210-218, 2009.

SHIVELY,T.E.; WILLIAMS, G.L. Patterns of tonic luteinizing hormone release and ovulation frequency in suckled anestrous beef cows following varyingintervals of temporary weaning. Domestic Animal Endocrinology. V.6, p.379-387, 1989.

WALTERS, D.L.; SMITH,M.F.; HARMS,P.G. Effect of steroids and 48 hr calf removal on serum luteining hormone concentrations in anestous beef cows. Theriogenology. v.18, P. 349-356, 1982.

WILLIAMS, G.L.; GRIFFITH, M.K. Sensory and behavioural controlo $f$ gonadotrophin secretion during sucklingmediated anovulation in cows. Journal of Reproduction and Fertility. v.49, p.463475, 1995.

YAVAS, Y.; WALTON, J.S. Postpartum acyclicity in suckled beef cows: a review. Theriogenology. v.54,p. 25-55,2000. 